\title{
Reciclagem de Materiais Plásticos: A Importância da Identificação Correta
}

\author{
Leda Coltro, Bruno F. Gasparino, Guilherme de C. Queiroz
}

CETEA, ITAL

\begin{abstract}
Resumo: Muitos produtos de material plástico apresentam código de identificação (normalmente um número de 1 a 7 dentro de um triângulo de três setas e sob o mesmo uma abreviatura) indicando o tipo de plástico do qual o produto é feito para auxiliar sua separação e posterior reciclagem e revalorização, contribuindo com a recuperação dos materiais plásticos descartados com o resíduo sólido urbano. Como as embalagens têm rotatividade alta, é importante que as mesmas apresentem o símbolo de identificação da resina a fim de facilitar a cadeia de reciclagem do plástico. Neste trabalho, foi feito um levantamento de dados sobre os símbolos de identificação dos materiais plásticos em um total de 177 embalagens plásticas rígidas para o acondicionamento de diversos produtos alimentícios e não alimentícios disponíveis no mercado brasileiro. Apesar da norma brasileira ABNT NBR 13230 já ter 14 anos, há ainda heterogeneidade na identificação das embalagens plásticas. Somente cerca de $80 \%$ das embalagens avaliadas apresentaram o símbolo de identificação da resina. Além disso, em alguns casos até $40 \%$ das embalagens apresentaram a identificação do material de forma incorreta. Portanto, ainda existe informação errônea no mercado brasileiro sobre o tipo de material da embalagem plástica (incluindo ausência do símbolo de identificação), bem como falta de informação sobre o símbolo correto de identificação da resina, sendo que ambos os fatores prejudicam a cadeia de reciclagem do plástico.
\end{abstract}

Palavras-chave: Embalagens, plástico rígido, reciclagem, alimentos, higiene pessoal, produtos de limpeza.

\section{Plastic Materials Recycling: The Importance of the Correct Identification}

Abstract: Many plastic-based products show a resin identification code - usually a number from 1 to 7 inside a three-arrow triangle above a monogram - to identify the type of plastic used to make the product, for assisting in its separation and later recycling. In other words, to facilitate the recovery of plastics discarded with the municipal solid waste. Since packages have a high rotation, the presence of the resin identification code is important for the plastic recycling chain. In the present study, a survey was carried out with 177 rigid plastic packages for food and non-food products available in the Brazilian market. Although the identification code was a requirement from the ABNT NBR 13230 Brazilian standard issued 14 years ago, there are still problems with the resin identification code. Only approximately $80 \%$ of the evaluated packages had the resin identification code. Furthermore, up to $40 \%$ of packages had incorrect material identification. Therefore, misinformation still occurs in the Brazilian market concerning plastic packaging material type - including lack of resin identification code - as well as lack of information regarding the correct way to display the resin identification code in plastic packages. Both of these factors have negative effect on the plastic recycling chain.

Keywords: Packaging, rigid plastic, recycling, food, household, personal products.

\section{Introdução}

Muitos produtos feitos de materiais plásticos apresentam um código de identificação da resina, normalmente um número de 1 a 7 dentro de um triângulo de três setas e sob o mesmo uma abreviatura, cujo objetivo é indicar o tipo particular de plástico do qual o produto é feito. Este código normalmente é colocado na base do recipiente ou no verso da embalagem que contém o produto (no caso de material flexível). Os códigos de identificação têm por objetivo facilitar a recuperação dos recipientes plásticos descartados com o resíduo sólido urbano, uma vez que auxiliam sua separação e posterior reciclagem e revalorização.
Este sistema de código de identificação de resinas foi introduzido em 1988 pela Sociedade das Indústrias de Plásticos - Society of Plastics Indutry, Inc. (SPI) devido a uma solicitação dos recicladores. Portanto, o sistema de códigos do SPI foi desenvolvido para atender as necessidades dos recicladores e, ao mesmo tempo, forneceu aos fabricantes um sistema consistente e uniforme que passou a ser aplicado em todo o território americano.

Uma vez que os programas de reciclagem municipal tradicionalmente têm a embalagem como meta, no caso das embalagens plásticas rígidas, o sistema de códigos do SPI propiciou um meio de identificação do tipo de resina das garrafas e frascos plásticos normalmente encontrados no resíduo sólido urbano. O sistema de códigos impulsiona

Autor para correspondência: Leda Coltro, CETEA, ITAL, Av. Brasil 2880, Caixa Postal 139, CEP: 13073-001, Campinas, SP, Brasil. E-mail: ledacolt@ital.org.br 
o controle de qualidade na linha de separação de materiais plásticos nos recicladores, assegurando que o plástico reciclado seja o mais homogêneo possível ${ }^{[1]}$.

Apesar de ser mais utilizado para a identificação de embalagens plásticas, este sistema também deveria ser aplicado a outros produtos de plástico, tais como pastas, capas, envelopes, canetas, bandejas, cadeiras, bancos, vasos, peças de equipamentos eletrônicos, sistemas de acolchoamento e outros inúmeros produtos de plástico utilizados pela sociedade, com a finalidade de facilitar a logística reversa.

A logística reversa é a área da logística empresarial que tem a preocupação com os aspectos logísticos do retorno dos produtos, materiais e embalagens ao ciclo produtivo. A logística reversa foi bastante empregada há algum tempo atrás pelas indústrias de bebidas devido à utilização das garrafas retornáveis e seus engradados. A prática da logística reversa pode ser observada também nas indústrias de latas de alumínio e de papelão ondulado, onde se destaca o grande aproveitamento de matéria-prima reciclada. Outros exemplos são a reciclagem de plásticos, de papel, de pneus, a utilização de paletes, etc. Portanto, a logística reversa é uma etapa importante para o processo de reciclagem, uma vez que os materiais pós-consumo retornam aos diferentes centros produtivos na forma de matéria-prima.

O sistema de código de identificação de resinas do SPI serviu como base para a norma da ABNT NBR 13230 (1994) - "Simbologia indicativa de reciclabilidade e identificação de materiais plásticos" que atualmente está em processo de revisão ${ }^{[2]}$. Os símbolos de identificação dos materiais plásticos são apresentados na Figura 1. Os seis materiais identificados pelos símbolos são os plásticos que predominam no mercado. Na Tabela 1 são apresentados alguns exemplos de aplicação das resinas e os respectivos produtos feitos a partir de plástico reciclado.

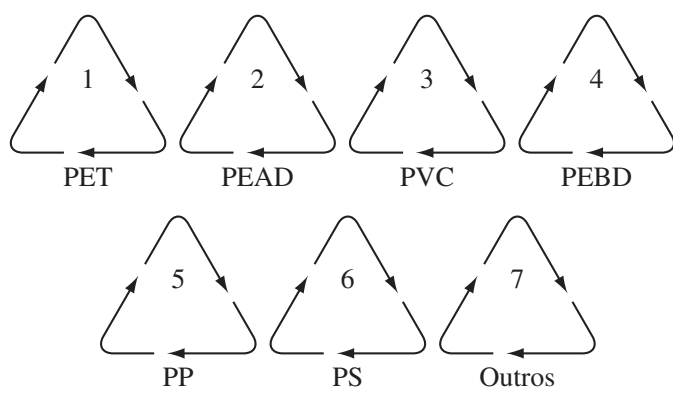
1- PET - Polietileno tereftalato
2 - PEAD - Polietileno de alta densidade
3 - PVC - Policloreto de vinila
4 - PEBD - Polietileno de baixa densidade
5 - PP - Polipropileno
6 - PS - Poliestireno
7 - Outros

Figura 1. Símbolos de identificação dos materiais plásticos segundo a norma ABNT NBR $13230^{[2]}$
No caso de produtos fabricados com polietileno linear de baixa densidade (PELBD), deve-se adotar o mesmo símbolo do PEBD (número 4) uma vez que estes materiais podem ser reciclados em um mesmo processo, sem separação prévia dos mesmos.

No caso de produtos fabricados com uma mistura de resinas, coextrusão e/ou coinjeção, os dois componentes principais da mistura devem ser indicados, por exemplo PEAD/PEBD (sob o triângulo) e 2/4 (dentro do triângulo).

O símbolo "7 - Outros" normalmente é empregado para produtos plásticos fabricados com policarbonato, ABS, poliamida, acrílicos ou uma combinação de diversas resinas e/ ou materiais. Neste caso, sugere-se também o uso da sigla do polímero abaixo do símbolo.

De acordo com a norma em referência, os caracteres indicativos da resina abaixo dos símbolos podem ser gravados em português, inglês ou ambos. Por este motivo, a norma apresenta a grafia de identificação das resinas nos dois idiomas.

Os plásticos rígidos devem ter o símbolo gravado nos respectivos moldes de fabricação. No caso de ser inviável tecnicamente a gravação do símbolo ou a alteração dos moldes, o símbolo deve ser impresso no fundo do recipiente plástico. Se o produto plástico for muito pequeno de modo que não permita a gravação e/ou impressão da simbologia completa, a norma recomenda que seja eliminada a gravação da abreviatura ficando obrigatória a identificação numérica dentro do triângulo.

Vale ressaltar que quando um mesmo molde é empregado para a fabricação de produtos plásticos iguais (copos, pratos, bandejas, etc.), porém com resinas diferentes, é aconselhável a adoção da impressão do símbolo no produto acabado ao invés da gravação do mesmo no molde. Adotando-se esta prática evita-se a indicação errônea do tipo de resina do produto, fato este já observado em produtos plásticos disponíveis no mercado brasileiro.

As dimensões dos símbolos apresentados na norma ABNT NBR 13230 ${ }^{[2]}$ são apenas orientativas, porém as proporções estabelecidas na norma devem ser obedecidas. Vale ressaltar que as dimensões devem facilitar ao máximo a leitura do mesmo e conseqüente separação do material na linha de triagem.

A norma não faz referência específica aos plásticos flexíveis, porém as embalagens flexíveis também devem adotar a simbologia desta norma. No caso de laminação e/ou coextrusão de diversos materiais para a fabricação da embalagem flexível deve-se indicar os dois componentes principais da estrutura. Esta identificação das resinas auxilia na reciclagem mecânica destas embalagens pois algumas embalagens multicamadas, tais como BOPP/BOPP, PEBD/adesivo/PA/adesivo/PEBD, $\mathrm{PP} /$ adesivo/EVOH/adesivo/PP, PET/adesivo/PEBD, PA/adesivo/PP, PVC/PE, PS/PE, etc. são viáveis para a reciclagem mecânica em processos específicos sem a necessidade de separação prévia das camadas da estrutura ${ }^{[4]}$.

No entanto, estruturas que contêm PVdC/OPP ou EVOH/PA apresentam dificuldades técnicas para a recicla- 
Tabela 1. Exemplos de aplicação e de reciclagem das resinas plásticas ${ }^{[1,3]}$.

\begin{tabular}{ll}
\multicolumn{1}{c}{ Aplicação } & \multicolumn{1}{c}{ Reciclagem } \\
\hline Resina & $\begin{array}{l}\text { Garrafas para refrigerante, água, óleo comestível, molho } \\
\text { para salada, anti-séptico bucal, xampu }\end{array}$ \\
fibra para carpete, tecido, vassoura, embalagem de produtos \\
dimpeza, acessórios diversos
\end{tabular}

gem mecânica devido a instabilidade térmica do PVdC e do EVOH frente aos respectivos componentes das estruturas. Por estes motivos é importante que as resinas sejam identificadas na embalagem. Desta forma, em muitos casos o símbolo mais indicado é o "7 - Outros" devido à combinação de muitas resinas e/ou materiais distintos (alumínio, por exemplo).

À princípio todos os plásticos podem tecnicamente ser submetidos à reciclagem mecânica, mas os plásticos que de fato são reciclados variam dependendo da área de utilização. Esta "seleção" dos materiais está relacionada com o valor econômico e o volume de material disponível para reciclagem $^{[5]}$.

A reciclagem energética (aproveitamento do valor calorífico do resíduo plástico) é uma opção de revalorização quando a reciclagem mecânica não é viável na prática (por motivos técnicos e/ou econômicos).

\section{Experimental}

Foi feito um levantamento de dados sobre os símbolos de identificação dos materiais plásticos em um total de 177 embalagens plásticas rígidas disponíveis no mercado brasileiro empregadas para o acondicionamento de diversos produtos alimentícios (75 amostras) e não alimentícios (higiene pessoal, limpeza doméstica e óleo combustível) (102 amostras). Este levantamento de dados foi feito no varejo em Campinas e na cidade de São Paulo, avaliando-se também a presença ou não do símbolo de identificação da resina nos rótulos destas embalagens. A seleção das embalagens para o levantamento de dados tomou por base o tipo de produto acondicionado, com ênfase para as embalagens plásticas rígidas e consi- derando a representatividade do produto acondicionado no mercado brasileiro.

\section{Resultados e Discussão}

A presença do símbolo de identificação da resina na embalagem não garante ou implica que a mesma é adequada para a reciclagem, mas certamente facilita a separação e sua reciclagem ou, no mínimo, permite que esta não venha a comprometer a qualidade de outras cadeias de reciclagem. A adequação da resina reciclada para uma determinada aplicação depende da demanda desta aplicação e da natureza da contaminação resultante do uso anterior da embalagem.

Além disso, as resinas virgens são fabricadas com propriedades específicas de acordo com as necessidades da aplicação final. Já o mercado de material reciclado deve ser tolerante a variações nas propriedades que ocorrem entre os diferentes grades de uma mesma resina que estão misturados no resíduo sólido urbano ${ }^{[3]}$.

Apesar da norma brasileira já contar com 14 anos de existência, ainda existe muita heterogeneidade na identificação das embalagens disponíveis no mercado brasileiro (Figura 2). Além do uso de símbolos não normalizados, também existem muitas embalagens plásticas sem a identificação da resina, somente com o triângulo de três setas indicativo de material reciclável, ponto verde (símbolo de material coletado pelo sistema alemão - DSD), além daquelas onde a impressão do prazo de validade, número de lote, etc. estão impressos sobre o símbolo de identificação da resina.

Não se deve confundir a identificação para a reciclagem com a rotulagem ambiental (série de normas ISO 14020). 


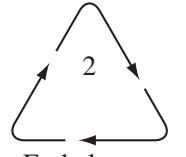

Embalagem reciclável
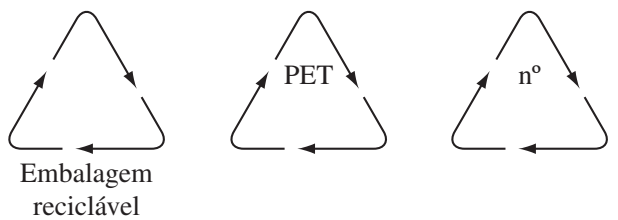

Figura 2. Símbolos de identificação (não padronizados) de algumas embalagens disponíveis no mercado brasileiro.

Considerando-se o primeiro desenho, no topo à esquerda, apresentado na Figura 2, a auto-declaração ambiental do tipo "embalagem reciclável" (rótulo ambiental do Tipo II, segundo a norma ISO 14021), está localizada erroneamente no espaço reservado para a identificação da resina de $\operatorname{PEAD}^{[6,7]}$.

Um exemplo de auto-declaração no formato correto é apresentado na Figura 3. Vale ressaltar que uma rotulagem ambiental do Tipo II, como a auto-declaração ressaltada pelo retângulo na Figura 3, deve ser verdadeira e verificável, ou seja, pelo Código de Defesa do Consumidor as empresas são obrigadas a fornecer informações publicitárias não enganosas ou abusivas de modo que, se for solicitado pelo consumidor, a empresa deve informar onde e como é feita a reciclagem da respectiva embalagem ${ }^{[8]}$.

Um resumo dos resultados obtidos do levantamento de dados sobre a identificação da resina em embalagens plásticas rígidas para produtos alimentícios e não alimentícios disponíveis no mercado é apresentado nas Tabelas 2 e 3. Observou-se que a maioria das embalagens apresentou o símbolo de identificação correto, porém o mesmo não foi observado com relação aos rótulos destas embalagens.

No caso dos produtos alimentícios, $83 \%$ das embalagens plásticas rígidas avaliadas apresentaram identificação da resina, sendo que $90 \%$ das embalagens de PET, PEAD e PP para estes produtos apresentaram identificação correta do material da embalagem.

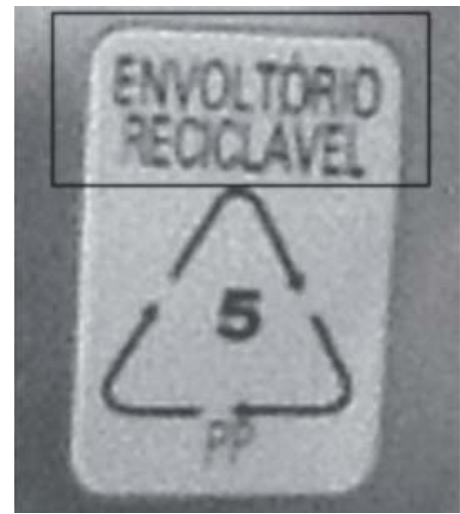

Figura 3. Símbolos de identificação e auto-declaração ambiental impressos de forma correta.
Apesar da apresentação correta do símbolo de reciclagem na embalagem de PP para catchup (Tabela 2), a identificação da resina poderia ser aprimorada pois os frascos de PP utilizados para o acondicionamento de catchup normalmente são multicamada, sendo as estruturas mais comuns de cinco ou seis camadas: $\mathrm{PP} / \mathrm{adesivo/EVOH/adesivo/PP} \mathrm{ou}$ $\mathrm{PP} /$ aparas/adesivo/EVOH/adesivo/PP. Nestas estruturas, o PP é usado como camada estrutural, além de apresentar boa resistência térmica. Já o EVOH é utilizado como material barreira ao oxigênio ${ }^{[9]}$. Assim, o símbolo de identificação mais adequado para esta embalagem é a indicação dos dois componentes principais da estrutura, nesse caso PP e EVOH, auxiliando na reciclagem mecânica destas embalagens, uma vez que são estruturas viáveis para a reciclagem mecânica em processos específicos sem a necessidade de separação prévia das camadas da estrutura.

No caso dos produtos não alimentícios (Tabela 3), 79\% das embalagens plásticas rígidas avaliadas apresentaram identificação da resina, sendo que a maioria das embalagens plásticas rígidas de PET, PEAD, PVC e PP apresentaram identificação correta do material da embalagem, enquanto que as embalagens de PEBD foram aquelas com maior índice de identificação incorreta da resina (Figura 4). Observouse também uma maior ausência de identificação do material nas embalagens para produtos não alimentícios (21\%) do que para produtos alimentícios $(17 \%)$.

A identificação dos materiais dos rótulos foi a mais complicada pois, ou não existia (a grande maioria dos produtos alimentícios e a totalidade dos não alimentícios não apresentaram identificação da resina dos rótulos) ou era confusa. Algumas vezes o rótulo apresentava identificação incorreta (uma das causas pode ser a tentativa de identificar o material do frasco no rótulo), o que pode confundir a cadeia de reciclagem.

Vale lembrar que os índices de reciclagem, assim como muitas taxas de reciclagem estabelecidas em legislações voltadas para a revalorização de plásticos, aplicam-se a todo o setor de materiais plásticos. Portanto, contribuir para a reciclagem dos materiais plásticos é fundamental para todo o setor envolvido.

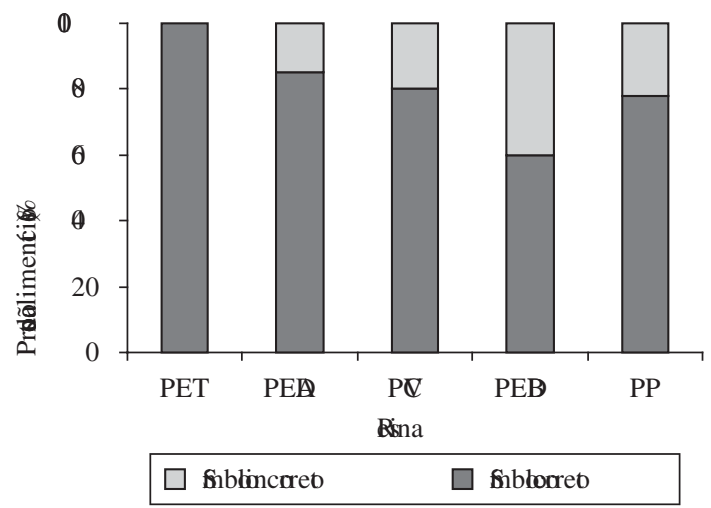

Figura 4. Porcentagem de produtos não alimentícios com símbolo de identificação correto e/ou incorreto na embalagem em função do tipo de resina plástica. 
Tabela 2. Símbolos de identificação utilizados em embalagens plásticas (e rótulos) para produtos alimentícios disponíveis no mercado.

\begin{tabular}{|c|c|c|c|c|c|}
\hline \multirow[t]{2}{*}{ Símbolo } & \multirow[t]{2}{*}{ Produto } & \multirow[t]{2}{*}{ Capacidade } & \multirow[t]{2}{*}{ N.A. } & \multicolumn{2}{|c|}{ Identificação } \\
\hline & & & & Frasco & Rótulo \\
\hline \multirow{18}{*}{ PET } & Adoçante & $65-100 \mathrm{~mL}$ & 4 & + & n.i. \\
\hline & Água & $510 \mathrm{~mL}$ & 5 & + & - \\
\hline & Catchup & $200-400 \mathrm{~g}$ & 2 & + & n.i. \\
\hline & Catchup & $200 \mathrm{~g}$ & 1 & + & - \\
\hline & Maionese & $500 \mathrm{~g}$ & 2 & + & - \\
\hline & Maionese & $500 \mathrm{~g}$ & 1 & + & n.i. \\
\hline & Mel & $200-340 \mathrm{~g}$ & 2 & + & n.i. \\
\hline & Mostarda & $180 \mathrm{~g}$ & 1 & + & n.i. \\
\hline & Óleo comestível & $900 \mathrm{~mL}$ & 2 & + & n.i. \\
\hline & Óleo comestível & $900 \mathrm{~mL}$ & 2 & - & - \\
\hline & Refrigerante & $600 \mathrm{~mL}$ & 1 & + & - \\
\hline & Refrigerante & $600 \mathrm{~mL}$ & 1 & - & - \\
\hline & Repositor energético & $500 \mathrm{~mL}$ & 1 & + & - \\
\hline & Repositor energético & $500 \mathrm{~mL}$ & 1 & + & n.i. \\
\hline & Sal & $100 \mathrm{~g}$ & 1 & + & n.i. \\
\hline & Suco & $500 \mathrm{~mL}$ & 1 & + & + \\
\hline & Vinagre & $750 \mathrm{~mL}$ & 3 & + & n.i. \\
\hline & Vinagre & $750 \mathrm{~mL}$ & 1 & + & - \\
\hline \multirow{7}{*}{ PEQ } & Adoçante & $65 \mathrm{~mL}$ & 1 & + & n.i. \\
\hline & Mostarda & $190 \mathrm{~g}$ & 1 & + & n.i. \\
\hline & Mostarda & $200 \mathrm{~g}$ & 1 & + & - \\
\hline & Achocolatado & $400 \mathrm{~g}$ & 1 & + & - \\
\hline & Cobertura para sobremesa & $230 \mathrm{~g}$ & 1 & + & - \\
\hline & Iogurte & $180-200 \mathrm{~g}$ & 4 & + & n.i \\
\hline & Iogurte & $180 \mathrm{~g}$ & 1 & - & + \\
\hline \multirow{10}{*}{$\underset{\mathrm{PP}}{S}$} & Catchup & $200 \mathrm{~g}$ & 1 & + & - \\
\hline & Mostarda & $200 \mathrm{~g}$ & 1 & + & - \\
\hline & Achocolatado & $210-400 \mathrm{~g}$ & 2 & + & n.i. \\
\hline & Achocolatado & $380 \mathrm{~g}$ & 1 & + & - \\
\hline & Cobertura para sobremesa & $240 \mathrm{~g}$ & 1 & + & n.i. \\
\hline & Cobertura para sobremesa & $320 \mathrm{~g}$ & 1 & - & n.i. \\
\hline & Doce de leite & $400 \mathrm{~g}$ & 4 & + & n.i. \\
\hline & Margarina & $500 \mathrm{~g}$ & 2 & + & sem rótulo \\
\hline & Requeijão & $220-250 \mathrm{~g}$ & 3 & + & n.i. \\
\hline & Tempero pronto & $300 \mathrm{~g}$ & 4 & + & n.i. \\
\hline \multirow{8}{*}{$\begin{array}{l}\text { Embalagens com } \\
\text { material não } \\
\text { identificado }\end{array}$} & Cobertura para sobremesa & $340 \mathrm{~g}$ & 1 & n.i. & - \\
\hline & Margarina & $500 \mathrm{~g}$ & 4 & n.i. & sem rótulo \\
\hline & Maionese & $500 \mathrm{~g}$ & 1 & n.i. & - \\
\hline & Maionese & $500 \mathrm{~g}$ & 1 & n.i. & n.i. \\
\hline & Mel & $450 \mathrm{~g}$ & 2 & n.i. & n.i. \\
\hline & Suco & $500 \mathrm{~mL}$ & 1 & n.i. & n.i. \\
\hline & Suco & $500 \mathrm{~mL}$ & 1 & n.i. & - \\
\hline & Sal & $100-300 \mathrm{~g}$ & 2 & n.i. & n.i. \\
\hline
\end{tabular}

N.A. = Número de amostras avaliadas; n.i. = Material não identificado; e +/- = Embalagem com identificação correta/Embalagem com identificação incorreta. 
Tabela 3. Símbolos de identificação utilizados em embalagens plásticas (e rótulos) para produtos não alimentícios disponíveis no mercado.

\begin{tabular}{|c|c|c|c|c|c|}
\hline \multirow[t]{2}{*}{ Símbolo } & \multirow[t]{2}{*}{ Produto } & \multirow[t]{2}{*}{ Capacidade } & \multirow[t]{2}{*}{ N.A. } & \multicolumn{2}{|c|}{ Identificação } \\
\hline & & & & Frasco & Rótulo \\
\hline \multirow{10}{*}{ PET } & Xampu & $350 \mathrm{~mL}$ & 1 & + & n.i. \\
\hline & Condicionador & $450 \mathrm{~g}$ & 1 & + & n.i. \\
\hline & Anti-séptico bucal & $250-500 \mathrm{~mL}$ & 3 & + & n.i. \\
\hline & Creme dental & $130 \mathrm{~g}$ & 1 & + & n.i. \\
\hline & Sabonete líquido & $250 \mathrm{~mL}$ & 1 & + & n.i. \\
\hline & Desinfetante & $500 \mathrm{~mL}$ & 3 & + & n.i. \\
\hline & Detergente & $500 \mathrm{~mL}$ & 1 & + & n.i. \\
\hline & Álcool gel & $500 \mathrm{~mL}$ & 1 & + & n.i. \\
\hline & Óleo para móveis & $200 \mathrm{~mL}$ & 1 & + & n.i. \\
\hline & Lustra móveis & $200-500 \mathrm{~mL}$ & 3 & + & n.i. \\
\hline \multirow{20}{*}{ PEQ } & Óleo combustível & $1 \mathrm{~L}$ & 3 & + & n.i. \\
\hline & Condicionador & $500 \mathrm{~g}$ & 1 & + & n.i. \\
\hline & Xampu & $350 \mathrm{~mL}$ & 2 & + & n.i. \\
\hline & Antitranspirante roll-on & $50-55 \mathrm{~mL}$ & 3 & + & n.i. \\
\hline & Antitranspirante roll-on & $65 \mathrm{~mL}$ & 1 & - & n.i. \\
\hline & Desodorante & $90 \mathrm{~mL}$ & 2 & - & n.i. \\
\hline & Gel após barba & $90 \mathrm{~g}$ & 1 & - & n.i. \\
\hline & Hidratante & $200-500 \mathrm{~mL}$ & 3 & + & n.i. \\
\hline & Protetor solar & $110-125 \mathrm{~mL}$ & 2 & + & n.i. \\
\hline & Repelente & $100 \mathrm{~mL}$ & 2 & + & n.i. \\
\hline & Detergente & $500 \mathrm{~mL}$ & 2 & + & n.i. \\
\hline & Multi-uso & $500 \mathrm{~mL}$ & 4 & + & n.i. \\
\hline & Álcool gel & $505 \mathrm{~g}$ & 1 & + & n.i. \\
\hline & Álcool líquido & $1 \mathrm{~L}$ & 2 & + & n.i. \\
\hline & Limpa vidros & $500 \mathrm{~mL}$ & 4 & + & n.i. \\
\hline & Cera auto brilho (piso) & $750 \mathrm{~mL}$ & 1 & + & n.i. \\
\hline & Cera auto brilho (piso) & $500-750 \mathrm{~mL}$ & 2 & - & n.i. \\
\hline & Alvejante & $500-750 \mathrm{~mL}$ & 2 & + & n.i. \\
\hline & Água sanitária & $1 \mathrm{~L}$ & 3 & + & n.i. \\
\hline & Amaciante & $1,8-2 \mathrm{~L}$ & 5 & + & n.i. \\
\hline \multirow[b]{3}{*}{$\mathrm{PQ}$} & Sabonete líquido & $150-200 \mathrm{~mL}$ & 2 & + & n.i. \\
\hline & Desinfetante & $500 \mathrm{~mL}$ & 2 & + & n.i. \\
\hline & Amaciante & $1 \mathrm{~L}$ & 1 & - & n.i. \\
\hline \multirow{4}{*}{ PEB } & Óleo combustível & $1 \mathrm{~L}$ & 1 & + & n.i. \\
\hline & Desodorante & $90 \mathrm{~mL}$ & 2 & + & n.i. \\
\hline & Antitranspirante bisnaga & $50 \mathrm{~g}$ & 1 & - & n.i. \\
\hline & Gel após barba & $120 \mathrm{~g}$ & 1 & - & n.i. \\
\hline \multirow{6}{*}{$\mathrm{PP}$} & Xampu & $300 \mathrm{~mL}$ & 1 & + & n.i. \\
\hline & Creme dental & $100 \mathrm{~g}$ & 1 & + & n.i. \\
\hline & Antitranspirante roll-on & $55 \mathrm{~g}$ & 2 & - & n.i. \\
\hline & Protetor solar & $200 \mathrm{~mL}$ & 1 & + & n.i. \\
\hline & Sabonete líquido & $250 \mathrm{~mL}$ & 2 & + & n.i. \\
\hline & Lustra móveis & $500 \mathrm{~mL}$ & 2 & + & n.i. \\
\hline \multirow{6}{*}{$\begin{array}{l}\text { Embalagens com material } \\
\text { não identificado }\end{array}$} & Anti-séptico bucal & $250-500 \mathrm{~mL}$ & 2 & n.i. & n.i. \\
\hline & Condicionador & $350-450 \mathrm{~g}$ & 2 & n.i. & n.i. \\
\hline & Creme dental & $100 \mathrm{~g}$ & 1 & n.i. & n.i. \\
\hline & Anti-transpirante bisnaga & $50-55 \mathrm{~g}$ & 3 & n.i. & n.i. \\
\hline & Desodorante & $90 \mathrm{~mL}$ & 2 & n.i. & n.i. \\
\hline & Gel após barba & $75 \mathrm{~g}$ & 1 & n.i. & n.i. \\
\hline
\end{tabular}


Tabela 3. Continuação...

\begin{tabular}{|c|c|c|c|c|c|}
\hline \multirow[t]{2}{*}{ Símbolo } & \multirow[t]{2}{*}{ Produto } & \multirow[t]{2}{*}{ Capacidade } & \multirow[t]{2}{*}{ N.A. } & \multicolumn{2}{|c|}{ Identificação } \\
\hline & & & & Frasco & Rótulo \\
\hline & Hidratante & $200 \mathrm{~mL}$ & 1 & n.i. & n.i. \\
\hline & Protetor solar & $120 \mathrm{~mL}$ & 2 & n.i. & n.i. \\
\hline & Sabonete líquido & $250 \mathrm{~mL}$ & 1 & n.i. & n.i. \\
\hline & Repelente & $100 \mathrm{~mL}$ & 1 & n.i. & n.i. \\
\hline & Detergente & $500 \mathrm{~mL}$ & 1 & n.i. & n.i. \\
\hline & Álcool gel & $480 \mathrm{~g}$ & 1 & n.i. & n.i. \\
\hline & Limpa vidros & $500 \mathrm{~mL}$ & 1 & n.i. & n.i. \\
\hline & Cera auto brilho (piso) & $750 \mathrm{~mL}$ & 1 & n.i. & n.i. \\
\hline & Alvejante & $650 \mathrm{~mL}$ & 1 & n.i. & n.i. \\
\hline
\end{tabular}

N.A. = Número de amostras avaliadas; n.i. = Material não identificado; e +/- = Embalagem com identificação correta/Embalagem com identificação incorreta.

\section{Conclusões}

Os resultados obtidos mostraram que:

s . O CASO DO PRODUIOS AIMENROIOS DAS EMBAA gens plásticas rígidas avaliadas apresentaram identificação da resina, sendo que $90 \%$ das embalagens de PET, PEAD e PP destes produtos apresentaram identificação correta do material da embalagem;

$s$. OCASODOPRODUOS NáOAIMENECOS DASEMBA lagens plásticas rígidas avaliadas apresentaram identificação da resina, sendo que $100 \%$ das embalagens de PET, 85\% das embalagens de PEAD, $80 \%$ das embalagens de PVC e 78\% das embalagens de PP apresentaram identificação correta do material. Por outro lado, as embalagens de PEBD foram aquelas que apresentaram a menor porcentagem de identificação correta da resina $(60 \%)$.

Portanto, ainda existe falta de informação no mercado brasileiro sobre o tipo de resina da embalagem plástica (ou seja, ausência do símbolo de identificação), bem como sobre o símbolo correto de identificação do material, sendo que ambos os fatores prejudicam a cadeia de reciclagem do plástico.

A falta de padronização do mercado dificulta grandemente a cadeia de reciclagem do plástico no Brasil. Assim, antes de gravar ou imprimir qualquer símbolo de identificação da resina plástica deve-se consultar a norma ABNT NBR 13230 e adotar o símbolo adequado ao produto plástico em questão.

Assim, a divulgação da norma ABNT NBR 13230 deve ser uma atividade constante das Associações e Instituições que trabalham com embalagens plásticas visando a máxima adequação deste setor e, assim, auxiliando a etapa de separação e posterior reciclagem dos plásticos.

\section{Agradecimentos}

Os autores agradecem à Rafael Comodo Gonçalves por sua colaboração neste estudo.

\section{Referências Bibliográficas}

1. EPIC - Environment and Plastics Industry Council. Plastics recycling made easier with resin codes. Special news $\&$ views report: different applications, different plastics. Mississauga, Ontario: p. 3-6, July 2001. Disponível em: <www.plastics.ca/epic>. Acesso: 06 set. 2006.

2. Associação Brasileira de Norma Técnicas - ABNT. Projeto de revisão NBR 13230: simbologia indicativa de reciclabilidade e identificação de materiais plásticos. Rio de Janeiro, 8p. (2006).

3. EPIC - Environment and Plastics Industry Council. SPI Plastic Container Coding System [s.d.] 2p. Disponível em: Disponível em: <www.plastics.ca/epic>. Acesso: 06 set. 2006.

4. Coltro, L. Embalagens Plásticas Flexíveis vs Meio Ambiente: Problema ou Solução? Curso "Embalagens Plásticas Flexíveis: Propriedades e Avaliação da Qualidade”. Campinas: CETEA/ITAL (Palestra) (2002).

5. Plastic New Zealand. The Plastic Identification Code - Bottoms up! Recycling plastic is easy at work and at home. Disnopível em:<www.plastics.org.nz>. Acesso em: 05 set. 2006.

6. International Organization for Standardization. ISO 14020: environmental labels and declarations - general principles. $2^{\text {nd }}$ ed. Genève: ISO, 5 p. (2000).

7. International Organization for Standardization. ISO 14021: environmental labels and declarations - type II self-declared environmental claims. Genève: ISO, 26 p. (2004).

8. Brasil. Código de Defesa do Consumidor. Lei 8.078/1990, de 11 de setembro de 1990. Dispõe sobre a proteção do consumidor e dá outras providências. Diário Oficial [da] República Federativa do Brasil, Brasília, DF, 12 set. 1990. Seção 3 (1990).

9. Antonio, J. T.; Sarantópoulos, C. I. G. L. \& Oliveira, P. A. P. L. V. - "Atomatados", in: Requisitos de Proteção de Produtos em Embalagens Plásticas Rígidas, cap. 7, (ed.), Léa M. de Oliveira (ed.), CETEA/ITAL, Campinas (2006).

Enviado: 20/06/07

Reenviado: 14/08/07

Aceito: 16/08/07 